\title{
Comparing Two Equations for Estimation of Kidney Function (Cockcroft-gault and Glomerular Filtration Rate Assessment in Liver Disease) for Vancomycin Dosing in Adult Liver Transplant Recipients: a Pilot, Randomized Clinical Trial
}

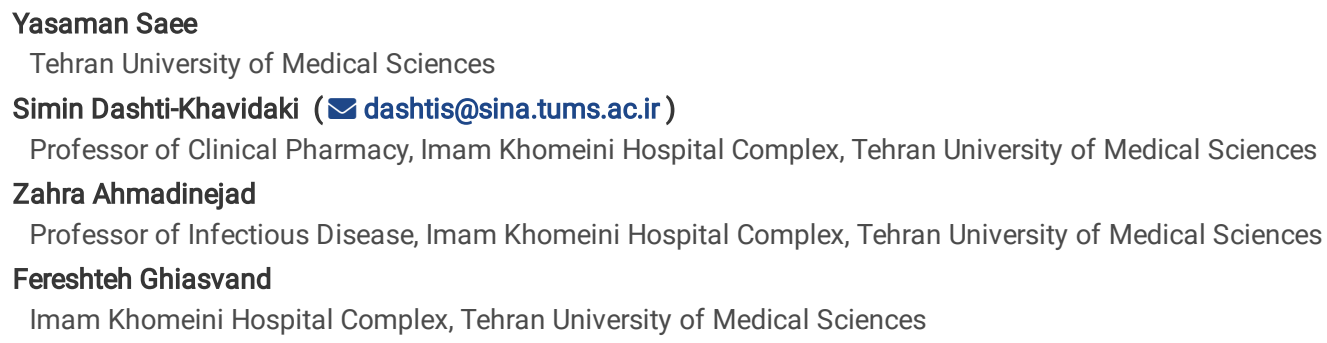

Version of Record: A version of this preprint was published at Pharmaceutical Sciences on December 26th, 2021. See the published version at https://doi.org/10.34172/PS.2021.79. 


\section{Abstract}

Background: In the setting of impaired liver function, estimation of glomerular filtration rate (GFR) using common creatinine based equations is inaccurate. Recently, Glomerular Filtration Rate Assessment in Liver Disease (GRAIL) model has been introduced for estimating GFR in liver transplantation. This study was conducted to compare vancomycin dose adjustment in liver transplant patients using Cockcroft-Gault (C-G) versus GRAIL method.

Methods: In this pilot, randomized clinical trial, adult liver transplant recipients who were candidate to receive intravenous vancomycin were enrolled. GFR and creatinine clearance were estimated using GRAIL model and C-G equation in the intervention and control arms, respectively and vancomycin maintenance doses were adjusted accordingly. At the steady state, peak and trough serum concentrations of vancomycin were collected for pharmacokinetic comparisons.

Results: Fifteen patients were enrolled in each arm of the study. Mean daily dose of vancomycin was estimated insignificantly lower for individuals in GRAIL arm than C-G group (1500.00 \pm 544.45 versus $1750.00 \pm 389.60 \mathrm{mg})$. The rate of patients who achieved the target vancomycin trough concentration was similar between the two study arms (20\%). Compared with C-G group, higher rate of patients in GRAIL arm experienced below-target vancomycin trough concentrations (40\% versus $13.3 \%$ ) and lower rate showed above target trough concentration ( $40 \%$ versus $66.7 \%$ ), although these differences did not reach statistical significance.

Higher rates of patients with at target and above target vancomycin AUC/MIC were seen in the C-G group compared with GRAlL group (40\% versus $26.7 \%$ and $53.3 \%$ versus $26.7 \%$, respectively), while individuals in the GRAIL arm represented significantly higher rate of below target vancomycin AUC/MIC than C-G arm (46.7\% versus $6.7 \%)(P=0.049)$. No differences in clinical outcomes were observed between the two groups.

Conclusion: Using GRAIL model for vancomycin dose selection may result in less percent of patients with at target AUC/MIC exposure compared to C-G method and expose more percent of patients at risk of vancomycin under dosing.

\section{Introduction}

Liver transplantation is the only life-saving and durable treatment option for patients with advanced liver disease. (1) A large number of liver transplant recipients has pre-existing impaired kidney function either independent of liver disease or as a sequel of advanced liver disease. (2) In addition, in early or late phase post transplantation, liver transplant recipients are at high risk of developing acute kidney injury (AKI) or chronic kidney disease due to various etiologies. $(3,4)$ Pre-transplant intrinsic kidney diseases or hepatorenal syndrome, renal hypo-perfusion, calcineurin inhibitors and comorbidities such as diabetes and hypertension are risk factors for developing or worsening renal dysfunction after liver transplantation. (5) Precise determination of kidney function is essential in liver transplant candidates because it affects decision on combined kidney and liver transplantation, estimate of medication dose adjustment, and modification of immunosuppression regimen. $(6,7)$

Although measurement of glomerular filtration rate (GFR) by clearance of exogenous markers such as inulin is considered the best way to assess kidney function, it is not routinely used in the clinical setting due to its cost and burden. (8) Serum creatinine (sCr) is a readily available test and has been widely used as an endogenous biomarker for GFR estimation. Renal function could be easily estimated by sCr-based equations such as Cockcroft-Gault (C-G), Modification of Diet in Renal Disease (MDRD-4, MDRD-6), and Chronic Kidney Disease Epidemiology Collaboration (CKD-EPI). These formulas were not specifically obtained from studies in liver transplant population or individuals with impaired liver function. (9-11) In the newest version of United States food and drug administration (FDA) guidance for industry, all these formulas have been accepted for estimation of renal function in pharmacokinetic studies of drugs. (12)

In the setting of impaired liver function, $\mathrm{sCr}$ could be detected falsely low or normal, therefore, estimating renal function using laboratory data such as sCr or blood urea nitrogen (BUN) by formulas not specifically derived in this population is not precise. (13) Limitations of sCr-based equations for estimating GFR in solid organ transplantation was evaluated in a meta-analysis. In that meta-analysis, $35 \%$ of the study population were liver transplant recipients. The most two accurate formulas were CKD-EPI and MDRD without any difference between them. Performance of MDRD and CKD-EPI were higher for patients with lower GFR and higher GFR, respectively. (14)

Recently, Glomerular Filtration Rate Assessment in Liver Disease (GRAIL) model has been introduced for estimating GFR, classification of kidney function and organ allocation in patients with liver diseases pre and post liver transplantation. GRAIL model has been designed from a 30-year, multi-center, cohort study of 3085 liver transplant patients. More than 12000 GFR samples were measured using urinary clearance of ${ }^{125}$-iothalamate at different time points pre and post liver transplantation. In this model some factors such as age, gender, race, sCr, BUN and albumin are used in order to calculate GFR. GRAIL model consists of various equations based on time points relative to transplant surgery and prediction of kidney function. Its online calculator is accessible at www.bswh.md/grail. (15) GRAIL has been compared with other estimated GFR equations. Although, all formulas overestimated the GFR, GRAIL method showed the least bias, the most precise and the highest accuracy in comparison to MDRD-4, MDRD-6 and CKD-EPI formulas for low GFR situations pre and post liver transplantation. It has been reported that GRAIL model had better performance in comparison to MDRD-4, MDRD-6 and CKD-EPI formulas even in patients with ascites. (15)

Although it seems that GRAIL model provides a better global estimate of renal function before and after liver transplantation, especially in the presence of kidney dysfunction, to the best of our knowledge no data has been published regarding its clinical use for drug dosing. This study was conducted to compare medication dose adjustment in liver transplant patients using C-G versus GRAIL method with therapeutic drug monitoring (TDM) of vancomycin as a drug which is predominantly cleared by kidneys.

\section{Material And Method}




\section{Study population}

This pilot study represents a single blinded, randomized, two parallel groups, clinical trial. Adult liver transplant recipients with stable kidney function (i.e. less than $0.3 \mathrm{mg} / \mathrm{dL}$ variations in $\mathrm{sCr}$ levels within 48 hours) who were candidate to receive intravenous vancomycin were evaluated for enrollment.

Patients with estimated GFR of less than $15 \mathrm{~mL} / \mathrm{min} / 1.73 \mathrm{~m}^{2}(16,17)$, re-transplantation or simultaneous kidney and liver transplantation(15), coadministration of oral vancomycin(18), change in vancomycin dose before obtaining drug levels(19), need for albumin administration within past two days (20), receiving medications which interact with $\mathrm{sCr}$ assay (such as cephalosporins, cimetidine, high dose vitamin $\mathrm{C}$ and catecholamines) (21-24), pregnant and lactating women were excluded from the study (25).

The study was conducted in the liver transplantation ward of Imam Khomeini Hospital Complex, affiliated to Tehran University of Medical Sciences, Tehran, Iran, during September 2019 to February 2021. All patients in this study received their organs from Iranian deceased donors based on Iranian national regulations for transplantation under the supervision of Ministry of Health and Medical Education. Based on these regulations, organs are not procured from prisoners. Organs were procured from Iranian National Tissue Bank at Imam Khomeini Hospital Complex.

Based on the center's protocol, liver transplant patients receive intravenous methylprednisolone during first five days after transplantation and then switch to oral prednisolone with tapering according to the center's protocol. Other maintenance immunosuppressive drugs include mycophenolate mofetil/sodium and a calcineurin inhibitor (tacrolimus or cyclosporine). All patients receive oral trimethoprim/sulfamethoxazole during 6 months post-transplantation for prevention of Pnemocystis jiroveci pneumonia, acyclovir for prophylaxis of Herpes zoster (if not candidate for chemoprophylaxis of cytomegalovirus) during first month after liver transplantation, Pre-emptive cytomegalovirus surveillance, and anti-fungal prophylaxis based on patient risk stratification (fluconazole and voriconazole for intermediate- and high-risk patients, respectively).

This project was approved by local ethics committee of Institute of Pharmaceutical Sciences, Tehran University of Medical Sciences (ID\# IR.TUMS.TIPS.REC.1398.003) and registered in Iranian registry of clinical trials (IRCT20100111003043N14) on 24/09/2019. All methods in this study were performed in accordance with the relevant guidelines and regulations including declaration of Helsinki. Participants signed informed consent form before recruitment.

\section{Intervention and data collection}

Patients' demographic data such as age, gender, height and weight, vital signs and laboratory data including sCr, serum albumin, BUN, liver enzymes, international normalized ratio (INR), bilirubin, blood cell counts, C-reactive protein (CRP), and microbial cultures as well as other related clinical data such as duration of hospitalization and treatment outcomes were recorded during the study period.

In the presence of suspected or documented resistant gram-positive cocci infection, the loading dose of vancomycin was administered by infectious disease specialist in a multi-disciplinary team and a specified clinical pharmacist was consulted to determine the maintenance dose of vancomycin in order to reach the serum trough level of $15-20 \mathrm{mg} / \mathrm{L}$. The clinical pharmacist assessed the patient's eligibility based on the inclusion and exclusion criteria for enrollment in the trial and equally allocated them into intervention (GRAIL) or control (C-G) group (allocation ratio of 1:1), according to blocked randomization list with block size of 4 which is created online by sealed envelope method. Participants were blind to groups assignment. Renal function was estimated using GRAIL model $\left(\mathrm{mL} / \mathrm{min} / 1.73 \mathrm{~m}^{2}\right)$ in the intervention arm and by $\mathrm{C}-\mathrm{G}$ equation $(\mathrm{mL} / \mathrm{min})$ using total body weight in the control arm. C-G calculated creatinine clearance was corrected for body surface area (BSA) $\frac{1.73 \times C G(\mathrm{ml} / \mathrm{min})}{B S A\left(\mathrm{~m}^{2}\right)}$ ) and expressed by $\mathrm{mL} / \mathrm{min} / 1.73 \mathrm{~m}^{2}$ unit. Equations are presented in Table 1. Proper vancomycin maintenance dose and interval were adjusted by the clinical pharmacist according to estimated GFR and total body weight as recommended by Golighty. (26) 
Table 1

Equations for GFR estimation and pharmacokinetic parameters

\begin{tabular}{|c|c|}
\hline $\mathrm{C}-\mathrm{G}(\mathrm{mL} / \mathrm{min})(9)$ & $([140-A g e($ year $)] \times$ totalbodyweight $(k g) \times[0.85$ iffemale $] /(\operatorname{Cr}(\mathrm{mg} / \mathrm{dL}) \times 72)$ \\
\hline \multirow[t]{3}{*}{$\begin{array}{l}\text { GRAIL model } \\
\left(\mathrm{mL} / \mathrm{min} / 1.73 \mathrm{~m}^{2}\right) \\
(15)\end{array}$} & $\operatorname{Constant}(i j) \times \operatorname{Gender}(i j)[$ iffemale $] \times \operatorname{Black}($ ij $)[$ ifPatientblack $] \times[\text { Age }(\text { year })]^{\text {Age }-\operatorname{Exp}(i j)} \times\left[\operatorname{Cr}\left(\frac{m g}{d L}\right)\right]^{C r-\operatorname{Exp}(i j)} \times[B U 1$ \\
\hline & $\mathrm{i}=0-1$ (low GFR trigger: 0 = predicted mGFR30+, 1 = predicted mGFR $₫ 30$ \\
\hline & $\mathrm{j}=1-6$ (timing: $1=$ pre liver transplant on waiting list, post liver transplant: $2=$ day $1-30,3=$ day $31-90,4=$ day $91-1$ year, $5=1-5$ yea \\
\hline \multirow[t]{5}{*}{$K(1 / h)(45)$} & $\ln ($ Cpeak/Ctrough $) / \Delta t$ \\
\hline & k: elimination rate constant \\
\hline & C peak: vancomycin peak concentration at steady-state \\
\hline & C trough: vancomycin trough concentration at steady-state \\
\hline & $\Delta \mathrm{t}$ : time interval between sampling peak and trough concentrations of a same dose \\
\hline \multirow{5}{*}{$\begin{array}{l}\mathrm{AUC}_{0-24} \\
(\mathrm{mg} . \mathrm{L} / \mathrm{h})(19)\end{array}$} & {$[t \times($ Cpeak + Ctrough $) / 2+($ Cpeak - Ctrough $) / k] \times 24 h r / \tau$} \\
\hline & t': infusion time \\
\hline & C peak: vancomycin peak concentration at steady-state \\
\hline & C trough: vancomycin trough concentration at steady-state \\
\hline & т: dose interval \\
\hline $\begin{array}{l}\text { New dose } \\
(\mathrm{mg} / \text { day })(19)\end{array}$ & targetconcentration / measuredconcentration $\times$ currentdose $(m g /$ day $)$ \\
\hline
\end{tabular}

At the steady state condition after the third maintenance dose of vancomycin, two blood samples as peak and trough concentrations of the fourth dose were collected. Peak level samples were obtained after distribution phase, i.e. 1 hour or 1.5-2.5 hours after completion of a 2-hour infusion or a 1-hour infusion of vancomycin, respectively. Trough level samples were drawn 30 minutes prior to the fifth dose of vancomycin. (19) Chemiluminescent microparticle immunoassay technique was used for quantitative measurement of vancomycin concentration via an Architect i1000SR analyzer (Abbott ${ }^{\circ}$; USA). Measurement range of this instrument was $0.24 \mathrm{mg} / \mathrm{L}$ to $100.00 \mathrm{mg} / \mathrm{L}$. (27)

A one-compartment model was assumed for vancomycin distribution phase to describe pharmacokinetic parameters. Participants' elimination rate constant (k) was computed using observed steady-state vancomycin peak and trough levels of the same fourth dose. Then the area under the concentration-time curve from 0 to 24 hours $\left(\mathrm{AUC}_{0-24}\right)$ was calculated. Pharmacokinetic formulas are presented in Table 1.

Considering local antimicrobial susceptibility patterns of Staphylococcus aureus in Imam-Khomeini Hospital Complex (28), minimum inhibitory concentration (MIC) of vancomycin was assumed $1 \mathrm{mg} / \mathrm{dL}$ and AUC/MIC was calculated. Vancomycin trough level and AUC/MIC were considered as at the target range at $15-20 \mathrm{mg} / \mathrm{L}$ and $400-600 \mathrm{mg} \times \mathrm{h} / \mathrm{L}$ respectively, below target at $<15 \mathrm{mg} / \mathrm{L}$ and $<400 \mathrm{mg} \times \mathrm{h} / \mathrm{L}$ and above target at $>20 \mathrm{mg} / \mathrm{L}$ and $>600 \mathrm{mg} \times \mathrm{h} / \mathrm{L}$, respectively. $(29)$ In either arm of the study, vancomycin new dose was calculated for patients whose fourth dose trough concentration was not in the target range by the formula presented in the Table 1. (19)

The primary outcome of this trial was comparison of percentages of patients reaching therapeutic pharmacokinetic targets in the steady state condition after initial vancomycin dose selection in either arm. Other primary end point was comparison of initial daily vancomycin dose between the two groups. Secondary objectives were comparisons of the duration of treatment with vancomycin, days of hospital stay, one-month mortality, resolution of fever or hypothermia, CRP reduction, improvement in leukocytosis or leukopenia and AKI due to vancomycin using Acute Kidney Injury Network classification (rise of serum Cr of $\geq 50 \%$ or increase of at-least $0.3 \mathrm{mg} / \mathrm{dL}$ in less than 48 hours or urine output of less than $0.5 \mathrm{cc} / \mathrm{kg} / \mathrm{h}$ for 6 consecutive hours in following days of vancomycin initiation after ruling out of other causes (30)) between study arms.

\section{Statistical analysis}

Quantitative variables were described by mean \pm standard deviation (SD) or median (interquartile rang (IQR)), which appropriate. Categorical variables were described using frequency and percentage. Normality of continues variables was assessed by Kolmogorov-Smirnov test. Univariate end points between arms were compared using Pearson chi-square test or Fisher exact test for categorical variables and t-test or Mann-Whitney test for continuous data. Vancomycin trough concentrations and AUC/MIC variabilities between treatment arms were compared with Levene's test. Log-rank test was used to compare patients' hospital length of stay between groups, accounting for the competing risk for death. For individuals with $\mathrm{C}-\mathrm{G}<60 \mathrm{~mL} / \mathrm{min}$ and $\geq 60 \mathrm{~mL} / \mathrm{min}$ at baseline, a stratified analysis was performed. For this analysis, Breslow-Day's test was used to assess the homogeneity of relationship between treatment arms and outcomes including vancomycin trough concentrations and AUC/MIC categories (below versus at or above target) across baseline C-G categories. Clinical outcomes based on vancomycin trough concentrations and AUC/MIC categories were compared using Fisher exact test for categorical outcomes and Kruskalwallis test for continues outcomes. Statistical analyses were conducted by SPSS, version 23 statistical software. P values less than 0.05 was considered statistically significant. 


\section{Results}

Fifteen patients in each arm completed the study (Fig. 1). As seen in Table 2, patients' characteristics and demographic data were comparable between the two study groups at baseline. Ninety percent of the patients were presented during the first year after transplantation. While the mean of the creatinine clearance based on C-G method were comparable between the study arms, GRAIL model showed a higher median [IQR] of kidney function estimation in the intervention than the control arm $\left(71.0[23.5,75.3]\right.$ versus $\left.25.9[19.5,29.1] \mathrm{ml} / \mathrm{min} / 1.73 \mathrm{~m}^{2} ; \mathrm{P}=0.051\right)$. 
Table 2

Patient characteristics and demographic data at baseline

\begin{tabular}{|c|c|c|c|c|}
\hline Characteristic & All (30) & GRAIL arm (15) & C-G arm (15) & $\mathbf{P}$ \\
\hline Age (years) & $46.17 \pm 12.71$ & $47.73 \pm 12.13$ & $44.60 \pm 13.49$ & 0.509 \\
\hline Gender & & & & 0.065 \\
\hline Female & 13(43.3) & $9(60.0)$ & $4(26.7)$ & \\
\hline Male & 17(56.7) & $6(40.0)$ & 11(73.3) & \\
\hline Weight (kg) & $63.87 \pm 10.54$ & $61.13 \pm 9.59$ & $66.60 \pm 11.05$ & 0.159 \\
\hline Height (cm) & $165.60 \pm 8.34$ & $163.20 \pm 8.57$ & $168.00 \pm 7.64$ & 0.117 \\
\hline $\operatorname{BSA}\left(\mathrm{kg} / \mathrm{m}^{2}\right)$ & $1.71 \pm 0.17$ & $1.65 \pm 0.17$ & $1.77 \pm 0.16$ & 0.065 \\
\hline $\mathrm{sCr}(\mathrm{mg} / \mathrm{dL})$ & $1.40 \pm 0.61$ & $1.38 \pm 0.77$ & $1.41 \pm 0.41$ & 0.884 \\
\hline BUN (mg/dL) & $33.73 \pm 15.97$ & $32.27 \pm 15.56$ & $35.20 \pm 16.77$ & 0.623 \\
\hline Albumin (g/dL) & $2.79 \pm 0.71$ & $2.80 \pm 0.63$ & $2.78 \pm 0.81$ & 0.94 \\
\hline Indication for liver transplantation & & & & 0.283 \\
\hline NASH & $9(31.0)$ & $3(21.4)$ & $6(40.0)$ & \\
\hline AlH & $9(31.0)$ & $7(50.0)$ & $2(13.3)$ & \\
\hline HBV & $1(3.4)$ & $1(7.1)$ & 0 & \\
\hline $\mathrm{HCV}$ & $1(3.4)$ & 0 & $1(6.7)$ & \\
\hline HBV-HDV & $1(3.4)$ & 0 & $1(6.7)$ & \\
\hline PSC & $4(13.8)$ & $1(7.1)$ & $3(20.0)$ & \\
\hline Cryptogenic & $2(6.9)$ & $1(7.1)$ & $1(6.7)$ & \\
\hline Acute liver failure & $1(3.4)$ & 0 & $1(6.7)$ & \\
\hline Liver metastasis & $1(3.4)$ & $1(7.1)$ & 0 & \\
\hline Time post liver transplant & & & & 0.327 \\
\hline Day $1-30$ & $16(53.3)$ & $7(46.7)$ & $9(60.0)$ & \\
\hline Day 31-90 & $6(20.0)$ & $5(33.3)$ & $1(6.7)$ & \\
\hline Day 91-year 1 & $5(16.7)$ & $2(13.3)$ & $3(20.0)$ & \\
\hline Year 1-5 & $3(10.0)$ & $1(6.7)$ & $2(13.3)$ & \\
\hline Calcineurin inhibitor agent & & & & 0.439 \\
\hline Tacrolimus & $20(66.7)$ & $9(60.0)$ & 11(73.3) & \\
\hline Cyclosporine & 10(33.3) & $6(40.0)$ & $4(26.7)$ & \\
\hline \multicolumn{5}{|l|}{ Renal function estimation } \\
\hline $\mathrm{C}-\mathrm{G}(\mathrm{mL} / \mathrm{min})$ & $66.10 \pm 26.43$ & $68.33 \pm 34.88$ & $63.87 \pm 14.79$ & 0.652 \\
\hline \multirow[t]{2}{*}{ GRAIL (mL/min/1.73m²) } & $45.02 \pm 27.33$ & $56.92 \pm 25.62$ & $33.11 \pm 24.22$ & 0.051 \\
\hline & $27.8[21.5,74.5]$ & $71.0[23.5,75.3]$ & $25.9[19.5,29.1]$ & $0.051^{\mathrm{a}}$ \\
\hline $\mathrm{C}-\mathrm{G}<60(\mathrm{~mL} / \mathrm{min})$ & 10(33.3) & $4(26.7)$ & $6(40.0)$ & 0.439 \\
\hline \multicolumn{5}{|c|}{$\begin{array}{l}\text { Note: Values for categorical variables are given as count (percentage); values for continuous variables, as mean } \pm \text { standard deviation or median } \\
\text { [interquartile range]. P-values come from Chi-square test for categorical variables and independent sample t-test or Mann-Whitney test for continues } \\
\text { variables. } \\
\text { a Data were skewed and thus median values are also presented in addition to mean values and Mann-Whitney test was used. } \\
\text { Abbreviations: BSA: body surface area; sCr: serum creatinine; BUN: blood urea nitrogen; NASH: non-alcoholic steatohepatitis; AlH: autoimmune hepatitis; } \\
\text { HBV: hepatitis B virus; HCV: hepatitis C virus; HDV: hepatitis D virus; PSC: primary sclerosing cholangitis; C-G: Cockcroft-Gault; GRAlL: glomerular filtration } \\
\text { rate assessment in liver disease. }\end{array}$} \\
\hline
\end{tabular}

Information about infectious disease, microbial cultures, and vancomycin dosages are presented in Table 3. Mean daily dose of vancomycin was lower for individuals in the GRAIL arm than C-G arm, although this difference did not reach statistical significance. 
Table 3

Infectious disease and antimicrobial information

\begin{tabular}{|c|c|c|c|c|}
\hline Characteristic & All $(n=30)$ & GRAIL arm(n= 15) & $C-G \operatorname{arm}(n=15)$ & $\mathbf{P}$ \\
\hline \multicolumn{5}{|l|}{ Suspected source of infection } \\
\hline Pulmonary & 13(43.3) & $6(40.0)$ & $7(46.7)$ & 0.713 \\
\hline Abdomen & $15(50.0)$ & $6(40.0)$ & $9(60.0)$ & 0.273 \\
\hline Skin and soft tissue & $4(13.3)$ & $4(26.7)$ & $0(0.0)$ & $0.100^{a}$ \\
\hline Unknown & $3(10.0)$ & $1(6.7)$ & $2(13.3)$ & $1.000^{a}$ \\
\hline \multicolumn{5}{|l|}{ Microbiology } \\
\hline Positive culture & $12(40.0)$ & $5(33.3)$ & $7(46.7)$ & 0.456 \\
\hline Gram + culture & $5(16.7)$ & $3(20.0)$ & 2(13.3) & $1.0^{\mathrm{a}}$ \\
\hline MRSA & $0(0.0)$ & $0(0.0)$ & $0(0.0)$ & - \\
\hline VSE faecium & $1(3.3)$ & $1(6.7)$ & $0(0.0)$ & $1.0^{\mathrm{a}}$ \\
\hline VSE faecalis & $1(3.3)$ & $0(0.0)$ & $1(6.7)$ & $1.0^{\mathrm{a}}$ \\
\hline VRE & $2(6.7)$ & $1(6.7)$ & $1(6.7)$ & $1.0^{\mathrm{a}}$ \\
\hline Vancomycin resistant streptococcus viridans & $1(3.3)$ & $1(6.7)$ & $0(0.0)$ & $1.0^{\mathrm{a}}$ \\
\hline Vancomycin daily dose & $1650.00 \pm 476.16$ & $1550.00 \pm 544.45$ & $1750.00 \pm 389.60$ & 0.367 \\
\hline \multicolumn{5}{|l|}{ Concomitant antibiotic } \\
\hline Meropenem & $27(90.0)$ & 13(86.7) & 14(93.3) & $1.0^{a}$ \\
\hline Ciprofloxacin & 10(33.3) & $4(26.7)$ & $6(40.0)$ & 0.439 \\
\hline Cefepime & $2(6.7)$ & $2(13.3)$ & $0(0.0)$ & $0.483^{a}$ \\
\hline Colistin & $4(13.3)$ & $3(20.0)$ & $1(6.7)$ & $0.598^{a}$ \\
\hline Levofloxacin & $1(3.3)$ & $0(0.0)$ & $1(6.7)$ & $1.0^{\mathrm{a}}$ \\
\hline \multicolumn{5}{|c|}{$\begin{array}{l}\text { Note: Values for categorical variables are given as count (percentage); values for continuous variables, as mean } \pm \text { standard deviation or median } \\
\text { interquartile range]. P-values come from Chi-square test or fisher's exact test for categorical variables and independent sample t-test or Mann-Whitney tes } \\
\text { for continues variables. } \\
\text { a Fisher's exact test. }\end{array}$} \\
\hline \multicolumn{5}{|c|}{$\begin{array}{l}\text { Abbreviations: MRSA; methicillin-resistant Staphylococcus aureus, VSE faecium; vancomycin-sensitive Enterococcus faecium, VSE faecalis; vancomycin- } \\
\text { sensitive Enterococcus faecium; VRE; vancomycin-resistant enterococci. }\end{array}$} \\
\hline
\end{tabular}

Number (percent) of patients who achieved the target vancomycin trough concentration was similar between the two study arms. Compared with C-G group, higher number of patients in GRAIL arm experienced below-target vancomycin trough concentrations and lower showed above target trough concentration, although these differences did not reach statistical significance.

Higher number of patients with at target and above target vancomycin AUC/MIC were seen in the C-G group compared with GRAIL group, while individuals in the GRAIL arm represented significantly higher rate of below target vancomycin AUC/MIC than C-G arm (Table 4). Vancomycin trough concentration and AUC/MIC showed lower variabilities in the C-G arm than GRAIL arm, although the differences were not significant (Fig. 2). 
Table 4

Vancomycin pharmacokinetic targets attainment and clinical outcomes based on study arms

\begin{tabular}{|c|c|c|c|c|}
\hline Characteristic & All (30) & GRAIL arm (15) & C-G arm (15) & $P$ \\
\hline \multicolumn{4}{|c|}{ Frequency of vancomycin target trough concentration attainment } & $0.276^{a}$ \\
\hline Below target & $8(26.7)$ & $6(40.0)$ & $2(13.3)$ & \\
\hline At target & $6(20.0)$ & $3(20.0)$ & $3(20.0)$ & \\
\hline Above target & 16(53.3) & $6(40.0)$ & $10(66.7)$ & \\
\hline \multicolumn{4}{|c|}{ Frequency of vancomycin target AUC/MIC attainment } & $0.049^{a}$ \\
\hline Below target & $8(26.7)$ & $7(46.7)$ & $1(6.7)$ & \\
\hline At target & 10(33.3) & $4(26.7)$ & $6(40.0)$ & \\
\hline Above target & $12(40.0)$ & $4(26.7)$ & 8(53.3) & \\
\hline \multicolumn{5}{|l|}{ Clinical outcomes } \\
\hline AKI due to vancomycin & $3(10.0)$ & $3(20.0)$ & $0(0.0)$ & $0.224^{a}$ \\
\hline Mortality & $2(6.7)$ & $2(13.3)$ & $0(0.0)$ & $0.483^{a}$ \\
\hline Hospital duration stay (days) & $24[16.75,72.75]$ & $21[16,102]$ & $24[19,63]$ & $0.728^{b}$ \\
\hline Vancomycin treatment duration (days) & $10[7,20.5]$ & $9[7,22]$ & $10[7,14]$ & $0.838^{c}$ \\
\hline CRP improvement ${ }^{\mathrm{e}}$ & $16(59.3)$ & $8(61.5)$ & $8(57.1)$ & $0.816^{d}$ \\
\hline Leukocytosis improvement ${ }^{f}$ & $10(47.6)$ & $3(33.3)$ & $7(58.3)$ & $0.387^{\mathrm{a}}$ \\
\hline Fever resolution ${ }^{g}$ & $19(100.0)$ & $9(100.0)$ & $10(100.0)$ & - \\
\hline \multirow{2}{*}{\multicolumn{5}{|c|}{$\begin{array}{l}\text { Note: Values for categorical variables are given as count (percentage); values for continuous variables, as mean } \pm \text { standard deviation or median } \\
\text { [interquartile range]. } \\
\text { a Fisher's exact test. }\end{array}$}} \\
\hline & & & & \\
\hline \multicolumn{5}{|c|}{ b Log-rank test for comparing length of stay, accounting for death as a competing risk. } \\
\hline \multicolumn{5}{|l|}{${ }^{\mathrm{c}}$ Mann-Whitney test. } \\
\hline \multicolumn{5}{|l|}{ d Pearson chi-square test } \\
\hline \multicolumn{5}{|c|}{ e Patients who achieved $\geq 50 \%$ reduction in amount of $\mathrm{CRP}$ at the end of the vancomycin treatment duration. } \\
\hline \multicolumn{5}{|c|}{ f Patients who achieved resolution or improvement in leukocytosis within 48 to 72 hours after initiation of vancomycin treatment. } \\
\hline \multicolumn{5}{|c|}{${ }^{g}$ Patients who achieved resolution of fever within 48 to 72 hours after initiation of vancomycin treatment. } \\
\hline \multicolumn{5}{|c|}{ Abbreviations: AKl; acute kidney injury, CRP; C reactive protein. } \\
\hline$c d$ & & & & \\
\hline
\end{tabular}

When all patients were stratified according to the estimation of their baseline kidney function using $\mathrm{C}-\mathrm{G}$ formula $(<60 \mathrm{~mL} / \mathrm{min}$ versus $\geq 60 \mathrm{~mL} / \mathrm{min})$, the effect of study group (GRAlL versus C-G arm) on distribution of vancomycin trough concentration was not modified by kidney function estimation (Fig. 3 ; $P=0.080$ ), but the effect of study group on vancomycin AUC/MIC distribution was modified (Fig. $3 ; P=0.011$ ). Among individuals with baseline $\mathrm{C}-\mathrm{G} \geq 60 \mathrm{~mL} / \mathrm{min}$ ( $\mathrm{n}=$ 20 ), patients in GRAIL arm showed higher rate of below target (63.6\% versus $0.0 \%)$, lower rate of at target (27.3\% versus $44.4 \%)$, and lower rate of above target (9.1\% versus $55.6 \%)$ AUC/MIC.

Clinical and laboratory outcomes including 4-week mortality, vancomycin treatment duration, time to fever resolution, and CRP or leukocytosis improvement were similar between the study arms $(P>0.05)$. Three patients $(10 \%)$ experienced vancomycin-induced AKI. They were in the GRAIL arm and had AUC $0-24$ of more than $880 \mathrm{mg} \times \mathrm{h} / \mathrm{L}$. Only hospital stay duration did significantly differ between patients who attained the target pharmacokinetic indices and who did not $(\mathrm{P}<0.001)$ (Table 4).

\section{Discussion}

Due to inaccuracy of conventional estimating GFR formulas for assessment of kidney function in liver transplant recipients and in patients with liver dysfunction and subsequent effects on medication dosing in the presence of impaired renal function, we designed this pilot study to evaluate the competence of GRAIL model, which is specifically designed for estimating kidney function in liver transplant patients, for drug dosing. 
This study exhibited that compared with C-G formula (the conventional, routine method for estimating kidney function), utilization of GRAIL model for estimation of GFR in adult liver transplant patients in order to selecting vancomycin maintenance dose resulted in more percent of patients in under dosing condition and below target vancomycin exposure, while using C-G method cause more individuals with AUC/MIC target attainment. However, vancomycin trough concentration was statistically comparable between the two study groups. Based on the latest guideline of the American Society of Health-System Pharmacists, the Infectious Diseases Society of America, and the Society of Infectious Diseases Pharmacists regarding therapeutic drug monitoring of vancomycin for serious methicillin-resistant Staphylococcus aureus infections, AUC/MIC has been introduced as the best

pharmacokinetic/pharmacodynamics index while implementation of vancomycin trough level alone for predicting of pharmacokinetic properties is associated with less accuracy.(29) Therefore, using GRAIL model for estimating kidney function and vancomycin dosing may put patients at the risk of under-dosing and perhaps treatment failure. Although clinical outcomes and adverse effects were comparable between the two study arms, this study was not sufficiently competence to determine these secondary outcomes because there are various confounding factors that influence these end points.

Administrating appropriate dose of medications is important to prevent treatment failure or developing adverse effects. Since vancomycin is a critical antibiotic for treatment of resistant gram positive infections while having narrow therapeutic index, vancomycin dosage adjustment should be performed individually using TDM. (31) Vancomycin is predominantly cleared unchanged by renal route and there is correlation between its body clearance and creatinine clearance so its primary dose selection is based on baseline kidney function. (32)

Although evaluation of kidney function is crucial in pre- and post- liver transplantation periods, contemporary equations have limited precision and have not been validated in this population. (33) Shaffi and colleagues in a systematic review compared sCr-based equations for estimating of kidney function in solid organ transplantation. One third of these patients were liver transplant recipients. They revealed that CKD-EPI and MDRD-4 may estimate GFR better than other formulas. CKD-EPI has better performance for GFR $\geq 60 \mathrm{~mL} / \mathrm{min} / 1.73 \mathrm{~m}^{2}$, while MDRD-4 better performs for GFR $<60 \mathrm{~mL} / \mathrm{min} / 1.73 \mathrm{~m}^{2}$. (14) Gonwa and colleagues compared several formulas including C-G, MDRD-4, MDRD-5, MDRD-6, and Nankivell with measured GFR using ${ }^{125}$-iothalamate in different phases relative to liver transplantation (pre-transplant, 3 months, 1 year and 5 years post transplantation). In pre-transplant phase, MDRD equation showed the greatest precision with limited accuracy, while all but Nankivell equations underestimated GFR. MDRD formulas showed better bias, precision, and accuracy at 1 year and 5 years after transplantation. This study revealed that using equations, kidney function is under estimated at GFR $\geq 40 \mathrm{~mL} / \mathrm{min} / 1.73 \mathrm{~m}^{2}$ and over estimated at GFR $<40 \mathrm{~mL} / \mathrm{min} / 1.73 \mathrm{~m}^{2}$. (33)

Based on 2020 FDA guidance for industries, pharmaceutical companies can apply any accepted formulas for either estimation of GFR (including MDRD and CKD-EPI) or estimation of creatinine clearance (including C-G using total body weight, ideal body weight, or adjusted body weight in over-weight and obese patients) in order to design drugs pharmacokinetic studies in patients with kidney dysfunction. GRAIL model has not yet been introduced by FDA for such studies. (12)

For a large number of medications, the C-G formula has been used for dose adjustment in pharmacokinetic studies in the presence of kidney dysfunction. Although it has been shown that GRAIL model results in a better estimation of kidney function in liver transplantation particularly in the case of impaired renal function, it has not yet been examined that if it could provide a better estimation for dosing of renally-eliminated medications. (15)

To the best of our knowledge this pilot, randomized, clinical trial was the first pharmacokinetic study which applied GRAIL model for dose calculation of vancomycin and compared it with C-G method. Although GRAIL model is specified for renal function assessment in liver transplant recipients, it seems that its implementation for vancomycin dose adjustment increases the frequency of below target vancomycin exposure and subsequent probable antimicrobial treatment failure.

Low hepatic production of creatinine, low skeletal muscle mass, increased tubular secretion of creatinine and interference of elevated bilirubin with creatinine assay in patients with impaired liver function, makes the serum creatinine an unreliable marker for assessing renal function in liver transplant patients; however, it is commonly employed in clinical practice due to ease and low cost of its assessment. $(34,35)$ Employing both sCr and cystatin $\mathrm{C}$ as appropriate GFR markers in one equation for estimation of renal function such as CKD-EPI creatinine-cystatin C equation have shown better performance than contemporary sCr-based equations such as C-G and MDRD methods or cystatin C-based equations in cirrhotic patients particularly for patients with GFR of less than $60 \mathrm{~mL} / \mathrm{min} / 1.73 \mathrm{~m}^{2}$. (36) Although its performance is better in non-cirrhotic patients, Cystatin C have not been tested for designing GRAlL model.

Few studies have compared the outcomes of using different equations for renal function estimation on vancomycin pharmacokinetic and dosing. Frazee and colleagues in a quality improvement project selected vancomycin dose based on two different kidney function estimation equations (C-G and CKD-EPI creatinine-cystatin C) in critically ill patients. They found that attainment of target trough concentration was 2 folds higher in CKD-EPI creatinine-cystatin C arm compared to C-G arm. (37) However, our study revealed that using the conventional C-G method for vancomycin dosing resulted in more pharmacokinetic index attainment compared to newly designed GRAIL model.

Taber and colleagues in a retrospective study on adult liver transplant patients compared estimated versus measured creatinine clearance and showed that $\mathrm{sCr}$ is not a suitable marker for estimating creatinine clearance and vancomycin pharmacokinetic properties in this population. (38) The results of our study is fairly consistent with the result of this study. Our project disclosed that more than $50 \%$ of patients did not reach the target pharmacokinetic indices in both arms of the study and it seems that none of these $\mathrm{sCr}$ based formulas performed adequately for estimating kidney function and drug dose selection.

An AUC/MIC of 400-600 mgxh/L creates a balance between vancomycin exposure-effect and its exposure-toxicity. (29) The rate of vancomycin-induced AKI has been reported to be in the range of 5 to $43 \%$ and it usually occurs after 72 hours of vancomycin initiation. Small number of studies have revealed that vancomycin trough concentrations of more than $15-20 \mathrm{mg} / \mathrm{L}$ and AUC values of more than 650 to $1300 \mathrm{mg} \times \mathrm{h} / \mathrm{L}$ are associated with increased AKI prevalence. Coadministration of other nephrotoxic medications (such as furosemide, amphotericin, amikacin, vasopressors and intra venous dye contrasts which may be 
needed to be administered after liver transplantation) could increase the risk of developing AKI due to vancomycin. $(29,39,40)$ In the present study, 3 patients (10\%) who had AUC of more than $880 \mathrm{mg} \times \mathrm{h} / \mathrm{L}$ showed vancomycin-induced AKI which is similar to previous mentioned investigations.

The same as former studies, our project represented that patients had low serum albumin concentration. Low serum albumin is prevalent in post liver transplant patients and is associated with increased risk of AKI. (41) Limited number of studies have investigated the effects of liver function adequacy on vancomycin pharmacokinetics. $(42,43)$ Mizuno and colleagues in a retrospective study on elderly patients showed that severe low serum albumin (defined as serum albumin of less than $2.5 \mathrm{~g} / \mathrm{dL}$ ) was associated with more percent of patients with AUC of more than $450 \mathrm{mg} \times \mathrm{h} / \mathrm{L}$, longer vancomycin half-life and more incidence of nephrotoxicity.(44) Using serum albumin for calculating eGFR by GRAIL model may cause limitations for applying this method of GFR estimation for all liver transplant patients, especially in early phase after the surgery that albumin preparation is needed to be administered in some patients.

This study suffers some limitations. It was a pilot study with small number of patients. Further studies with larger sample size and also for dosing of other drugs with available TDM are needed to determine if GRAIL model is a suitable method for dose selection in liver transplant patients to maximize the efficacy and prevent medications toxicity. Lack of measuring GFR as the gold standard method for kidney function evaluation is another limitation of the present study.

In conclusion, although GRAIL model for assessing kidney function has been specifically derived from liver transplant recipients, using this method for vancomycin dose selection may result in less percent of patients with at target AUC/MIC exposure compared to C-G method and expose more percent of patients at risk of vancomycin under dosing and probable subsequent treatment failure.

\section{Abbreviations}

AKI: acute kidney injury

AUC: area under the concentration-time curve

BSA: body surface area

BUN: blood urea nitrogen

C-G: Cockcroft-Gault

CKD-EPI: Chronic Kidney Disease Epidemiology Collaboration

CRP: C-reactive protein

FDA: United States food and drug administration

GFR: glomerular filtration rate

GRAIL: Glomerular Filtration Rate Assessment in Liver Disease

INR: international normalized ratio

MDRD: Modification of Diet in Renal Disease

MIC: minimum inhibitory concentration

sCr: serum creatinine

TDM: therapeutic drug monitoring

\section{Declarations}

Ethics approval and consent to participate: This project was approved by local ethics committee of Institute of Pharmaceutical Sciences, Tehran University of Medical Sciences (ID\# IR.TUMS.TIPS.REC.1398.003) and registered in Iranian registry of clinical trials (IRCT20100111003043N14) on 24/09/2019. All methods in this study were performed in accordance with the relevant guidelines and regulations including declaration of Helsinki. Participants signed informed consent form before recruitment.

Consent for publication: All participants signed consent form for study publication anonymously. All authors consent for publication.

Availability of data and materials: All data has been included in the manuscript. Patients' level data would be available by sending request to corresponding author.

Competing interests: All authors declare no competing interest.

Funding: This manuscript was the result of a Clinical Pharmacy thesis supported by Tehran University of Medical Sciences. There is no funding support.

Authors' contributions:

Page 10/14 
All have read and approved the manuscript.

Y.S.: Selected the patients, collected clinical and laboratory data, analyzed data, drafted and reviewed the manuscript.

S.D-K.: Conceptualized and designed the study, supervised research process, interpreted the data, drafted and finalized the manuscript.

Z.A.: Clinically assessed the patients, gathered clinical data, reviewed the manuscript.

F.G.: Clinically assessed the patients, gathered clinical data, reviewed the manuscript

\section{Acknowledgement:}

Authors thank all nursing staffs of our liver transplant team, especially Mrs. Leila Jahan for their valuable help.

\section{References}

1. Duffy JP, Kao K, Ko CY, Farmer DG, McDiarmid SV, Hong JC, et al. Long-term patient outcome and quality of life after liver transplantation: analysis of 20year survivors. Ann Surg. 2010;252(4):652-661. doi: 10.1097/SLA.0b013e3181f5f23a.

2. Cullaro G, Verna EC, Lee BP. Chronic kidney disease in liver transplant candidates: a rising burden impacting post-liver transplant outcomes. Liver Transpl. 2020;26(4):498-506. doi: 10.1002/It.25694.

3. Barri YM, Sanchez EQ, Jennings LW, Melton LB, Hays S, Levy MF, et al. Acute kidney injury following liver transplantation: definition and outcome. Liver Transpl. 2009;15(5):475-483. doi: 10.1002/lt.21682.

4. Ojo AO, Held PJ, Port FK, Wolfe RA, Leichtman AB, Young EW, et al. Chronic renal failure after transplantation of a nonrenal organ. N Engl J Med. 2003;349(10):931-940. doi: 10.1056/NEJMoa021744.

5. Durand F, Francoz C, Asrani SK, Khemichian S, Pham TA, Sung RS, et al. Acute kidney injury after liver transplantation. Transplantation. 2018;102(10):1636-1649. doi: 10.1097/TP.0000000000002305.

6. Burra P, Burroughs A, Graziadei I, Pirenne J, Valdecasas JC, Muiesan P, et al. EASL clinical practice guidelines: liver transplantation. J Hepatol. 2016;64(2):433-485. doi: 10.1016/j.jhep.2015.10.006.

7. Charlton M, Levitsky J, Aqel B, O'Grady J, Hemibach J, Rinella M, et al. International liver transplantation society consensus statement on immunosuppression in liver transplant recipients. Transplantation. 2018;102(5):727-743. doi: 10.1097/TP.0000000000002147.

8. Stevens LA, Coresh J, Greene T, Levey AS. Assessing kidney function-measured and estimated glomerular filtration rate. N Engl J Med. 2006;354(23):2473-2483. doi: 10.1056/NEJMra054415.

9. Cockcroft DW, Gault MH. Prediction of creatinine clearance from serum creatinine. Nephron. 1976;16(1):31-41. doi: 10.1159/000180580.

10. Levey AS, Bosch JP, Lewis JB, Greene T, Rogers N, Roth D. A more accurate method to estimate glomerular filtration rate from serum creatinine: a new prediction equation. Modification of Diet in Renal Disease Study Group. Ann Intern Med. 1999;130(6):461-470. doi: 10.7326/0003-4819-130-6199903160-00002.

11. Levey AS, Stevens LA, Schmid CH, Zhang YL, Castro AF, 3rd, Feldman HI, et al. A new equation to estimate glomerular filtration rate. Ann Intern Med. 2009;150(9):604-612. doi: 10.7326/0003-4819-150-9-200905050-00006.

12. Guidance for industry pharmacokinetics in patients with impaired renal function-study design, data analysis, and impact on dosing. Draft guidance 2020 [30 March 2021]. Available from: https://www.fda.gov/media/78573/download.

13. Papadakis MA, Arieff Al. Unpredictability of clinical evaluation of renal function in cirrhosis. Prospective study. Am J Med. 1987;82(5):945-952. doi: 10.1016/0002-9343(87)90156-2.

14. Shaffi K, Uhlig K, Perrone RD, Ruthazer R, Rule A, Lieske JC, et al. Performance of creatinine-based GFR estimating equations in solid-organ transplant recipients. Am J Kidney Dis. 2014;63(6):1007-1018. doi: 10.1053/j.ajkd.2014.01.436.

15. Asrani SK, Jennings LW, Trotter JF, Levitsky J, Nadim MK, Kim WR, et al. A model for glomerular filtration rate assessment in liver disease (GRAIL) in the presence of renal dysfunction. Hepatology. 2019;69(3):1219-1230. doi: 10.1002/hep.30321.

16. Matzke GR, Aronoff GR, Atkinson Jr AJ, Bennett WM, Decker BS, Eckardt K-U, et al. Drug dosing consideration in patients with acute and chronic kidney disease-a clinical update from Kidney Disease: Improving Global Outcomes (KDIGO). Kidney Int. 2011;80(11):1122-1137. doi: 10.1038/ki.2011.322.

17. Regal RE, Ren SP, Paige G, Alaniz C. Evaluation of vancomycin dosing in patients with cirrhosis: beginning de-liver-ations about a new nomogram. Hosp Pharm. 2018;54(2):125-129. doi: 10.1177/0018578718772266.

18. Cimolai N. Does oral vancomycin use necessitate therapeutic drug monitoring? Infection. 2020;48(2):173-182. doi: 10.1007/s15010-019-01374-7.

19. Duby J, Donnelley MA, Tasaka CL, H. HB. Vancomycin. In: Murphy JE, F.A.S.H.P, editors. Clinical pharmacokinetics. 6th ed. Bethesta, MD: American Society Health-System Pharmacists; 2016. p. 377-401.

20. Hahn RG, Lyons G. The half-life of infusion fluids: an educational review. Eur J Anaesthesiol. 2016;33(7):475-482. doi: 10.1097/EJA.0000000000000436.

21. Martinello F, da Silva EL. Ascorbic acid interference in the measurement of serum biochemical parameters: in vivo and in vitro studies. Clin Biochem. 2006;39(4):396-403. doi: 10.1016/j.clinbiochem.2005.11.011.

22. Saenger AK, Lockwood C, Snozek CL, Milz TC, Karon BS, Scott MG, et al. Catecholamine interference in enzymatic creatinine assays. Clin Chem. 2009;55(9):1732-736. doi: 10.1373/clinchem.2009.127373.

23. Swain RR, Briggs SL. Positive interference with the Jaffé reaction by cephalosporin antibiotics. Clin Chem. 1977;23(7):1340-1342. 
24. Hilbrands LB, Artz MA, Wetzels JF, Koene RA. Cimetidine improves the reliability of creatinine as a marker of glomerular filtration. Kidney Int. 1991;40(6):1171-1176. doi: 10.1038/ki.1991.331.

25. Bowers RD, Cooper AA, Wente CL, Wilson DT, Johnson SW, Drew RH. Evaluation of a vancomycin dosing nomogram in obese patients weighing at least 100 kilograms. Pharma Pract (Granada). 2018;16(3):1204. doi: 10.18549/PharmPract.2018.03.1204.

26. Golightly L, Teitelbaum I, Kiser T, Levin DA, Barber GR, Jones MA, et al., editors. Renal pharmacotherapy: dosage adjustment of medications eliminated by the kidneys. New York: Springer; 2013.

27. ARCHITECT i Vancomycin [package insert]. USA: Abbott; 2008.

28. Esmaeili A, salehi M, Makhdoomi N, Rajabi M, H. Ardakani Y, Namazi S. Evaluation of the association between trough and area under the curve to minimum inhibitory concentration ratio(AUC24/MIC) of vancomycin in infected patients with MRSA. Pharm Sci.

29. Rybak MJ, Le J, Lodise TP, Levine DP, Bradley JS, Liu C, et al. Therapeutic monitoring of vancomycin for serious methicillin-resistant Staphylococcus aureus infections: a revised consensus guideline and review by the American Society of Health-System Pharmacists, the Infectious Diseases Society of America, the Pediatric Infectious Diseases Society, and the Society of Infectious Diseases Pharmacists. Am J Health Sys Pharm. 2020;77(11):835-864. doi: 10.1093/ajhp/zxaa036.

30. Park SJ, Lim NR, Park HJ, Yang JW, Kim M-J, Kim K, et al. Evaluation of risk factors for vancomycin-induced nephrotoxicity. Int J Clin Pharm. 2018;40(5):1328-1334. doi: 10.1007/s11096-018-0634-8.

31. de Velde F, Mouton JW, de Winter BCM, van Gelder T, Koch BCP. Clinical applications of population pharmacokinetic models of antibiotics: challenges and perspectives. Pharmacol Res. 2018;134:280-288. doi: 10.1016/j.phrs.2018.07.005.

32. Eyler RF, Shvets K. Clinical Pharmacology of Antibiotics. Clin J Am Soc Nephrol. 2019;14(7):1080-1090. doi: 10.2215/CJN.08140718.

33. Gonwa TA, Jennings L, Mai ML, Stark PC, Levey AS, Klintmalm GB. Estimation of glomerular filtration rates before and after orthotopic liver transplantation: evaluation of current equations. Liver Transpl. 2004;10(2):301-309. doi: 10.1002/It.20017.

34. Beben T, Rifkin DE. GFR estimating equations and liver disease. Adv Chronic Kidney Dis. 2015;22(5):337-342. doi: 10.1053/j.ackd.2015.05.003.

35. Rognant N, Lemoine S. Evaluation of renal function in patients with cirrhosis: where are we now? World J Gastroenterol. 2014;20(10):2533-2541. doi: 10.3748/wjg.v20.i10.2533.

36. Mindikoglu AL, Dowling TC, Weir MR, Seliger SL, Christenson RH, Magder LS. Performance of chronic kidney disease epidemiology collaboration creatinine-cystatin C equation for estimating kidney function in cirrhosis. Hepatology. 2014;59(4):1532-1542. doi: 10.1002/hep.26556.

37. Frazee E, Rule AD, Lieske JC, Kashani KB, Barreto JN, Virk A, et al. Cystatin C-guided vancomycin dosing in critically ill patients: a quality improvement project. Am J Kidney Dis. 2017;69(5):658-666. doi: 10.1053/j.ajkd.2016.11.016.

38. Taber DJ, Fann AL, Malat G, Dupuis RE. Evaluation of estimated and measured creatinine clearances for predicting the pharmacokinetics of vancomycin in adult liver transplant recipients. Ther Drug Monit. 2003;25(1):67-72. doi: 10.1097/00007691-200302000-00010.

39. Aljefri DM, Avedissian SN, Rhodes NJ, Postelnick MJ, Nguyen K, Scheetz MH. Vancomycin area under the curve and acute kidney injury: a meta-analysis. Clin Infect Dis. 2019;69(11):1881-1887. doi: 10.1093/cid/ciz051.

40. Lodise TP, Patel N, Lomaestro BM, Rodvold KA, Drusano GL. Relationship between initial vancomycin concentration-time profile and nephrotoxicity among hospitalized patients. Clin Infect Dis. 2009;49(4):507-514. doi: 10.1086/600884.

41. Sang BH, Bang JY, Song JG, Hwang GS. Hypoalbuminemia within two postoperative days is an independent risk factor for acute kidney injury following living donor liver transplantation: a propensity score analysis of 998 consecutive patients. Crit Care Med. 2015;43(12):2552-2561. doi: 10.1097/CCM.0000000000001279.

42. Brown N, Ho DH, Fong KL, Bogerd L, Maksymiuk A, Bolivar R, et al. Effects of hepatic function on vancomycin clinical pharmacology. Antimicrob Agents Chemother. 1983;23(4):603-609. doi: 10.1128/AAC.23.4.603.

43. Brunetti L, Song JH, Suh D, Kim HJ, Seong YH, Lee DS, et al. The risk of vancomycin toxicity in patients with liver impairment. Clin Microbiol Antimicrob. 2020;19(1):13. doi: 10.1186/s12941-020-00354-2.

44. Mizuno T, Mizokami F, Fukami K, Ito K, Shibasaki M, Nagamatsu T, et al. The influence of severe hypoalbuminemia on the half-life of vancomycin in elderly patients with methicillin-resistant Staphylococcus aureus hospital-acquired pneumonia. Clin Interv Aging. 2013;8:1323-1328. doi: $10.2147 /$ CIA.S52259.

45. Morbitzer KA, Rhoney DH, Dehne KA, Jordan JD. Enhanced renal clearance and impact on vancomycin pharmacokinetic parameters in patients with hemorrhagic stroke. J Intensive Care. 2019;7(1):51. doi: 10.1186/s40560-019-0408-y.

\section{Figures}




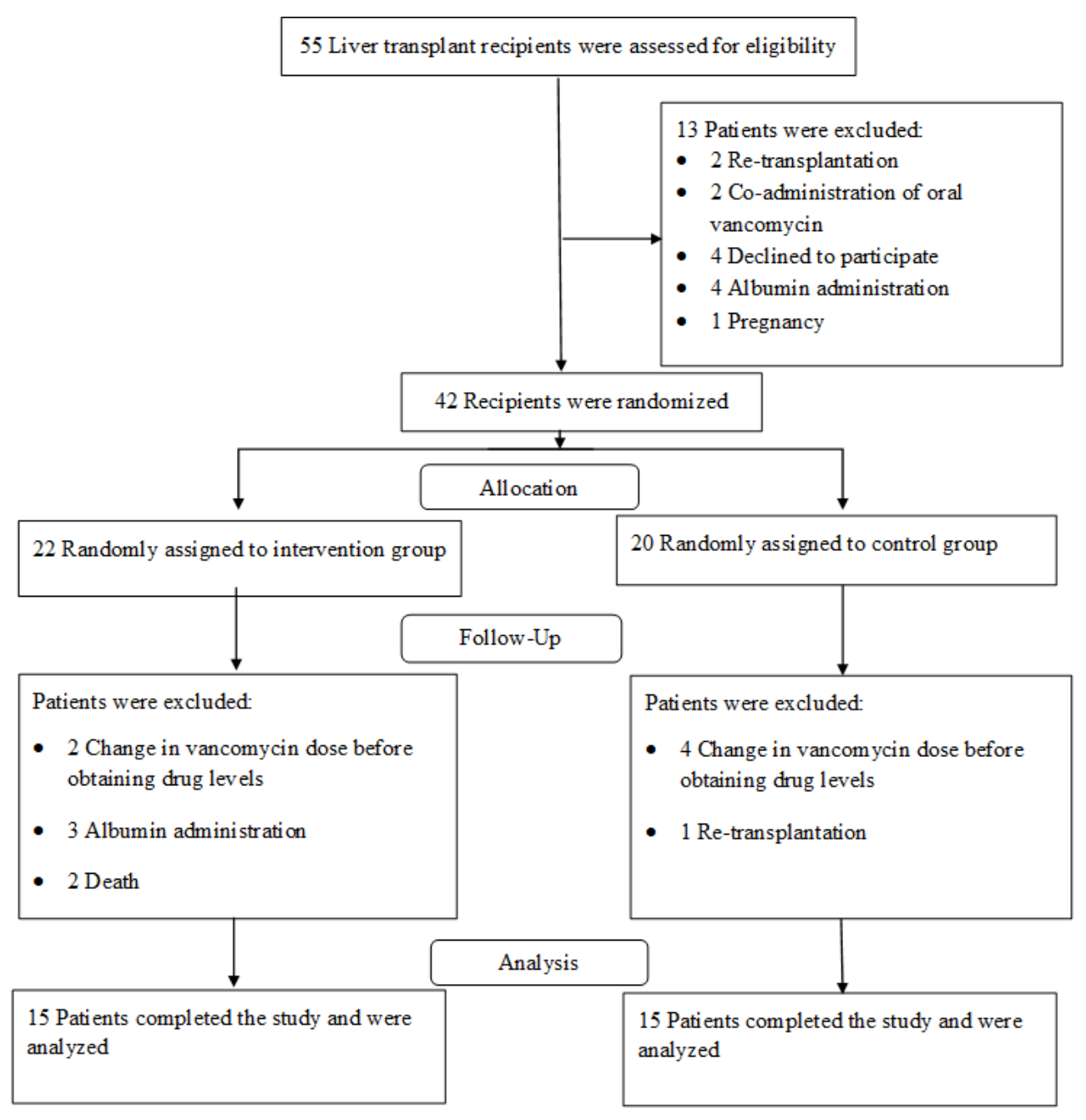

Figure 1

Patients enrollment flow chart.

a

b
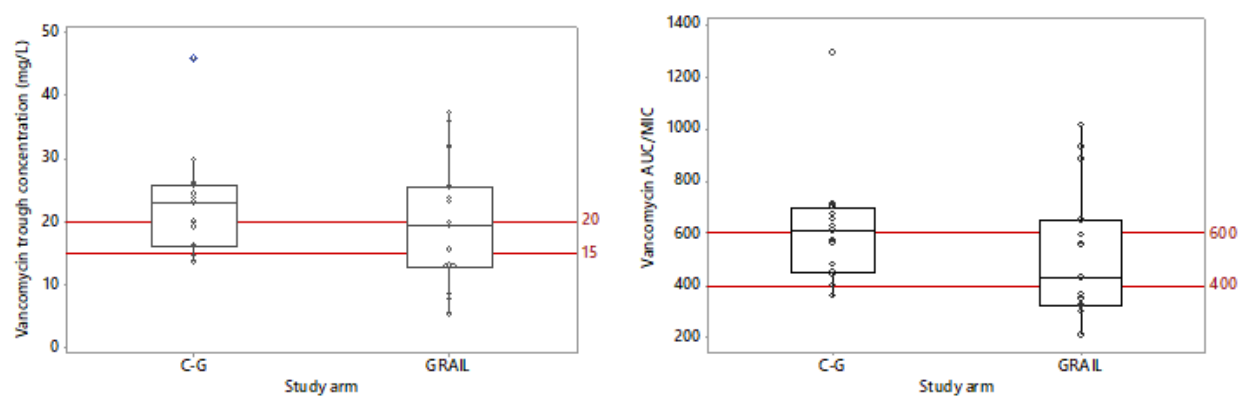

Figure 2

Vancomycin trough concentrations (a) and AUC/MIC (b) distributions according to the study groups. $(P>0.05)$ 


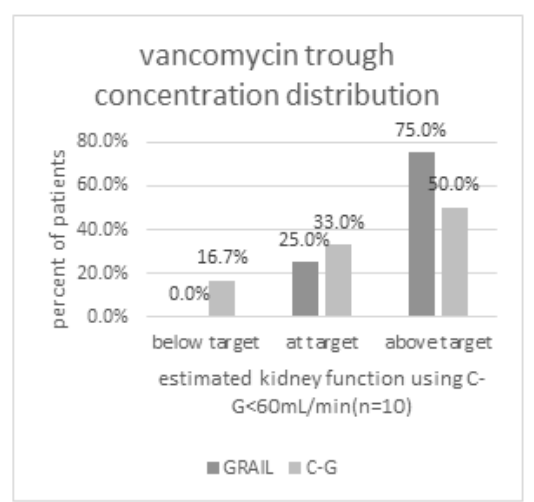

c

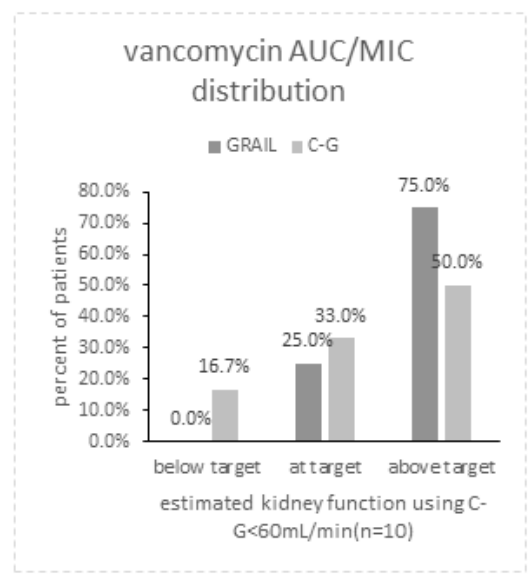

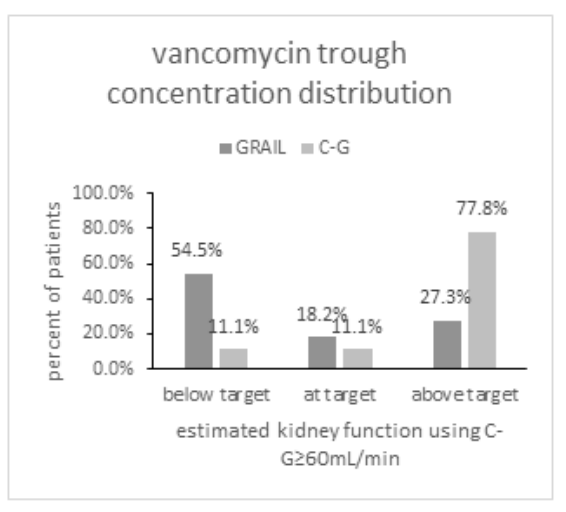

d

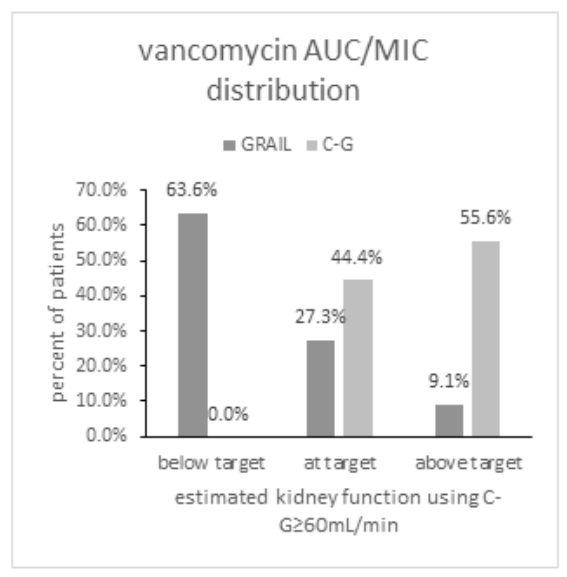

Figure 3

Stratified analysis based on kidney function estimation using C-G. The effect of study group on vancomycin trough concentration distribution was not modified by kidney function estimation using C-G at baseline ( $<60 \mathrm{~mL} / \mathrm{min}$ (a) versus $\geq 60 \mathrm{~mL} / \mathrm{min}(\mathrm{b}) ; \mathrm{P}=0.080$ ). The effect of study group on vancomycin AUC/MIC distribution was modified by kidney function estimation using C-G at baseline ( $<60 \mathrm{~mL} / \mathrm{min}$ (c) versus $\geq 60 \mathrm{~mL} / \mathrm{min}(\mathrm{d}) ; P=0.011)$. 